\title{
On Hadronic Models for the Anomalous $\gamma$-ray Emission Component in GRB 941017
}

\author{
Charles D. Dermer* and Armen Atoyan ${ }^{\dagger}$ \\ ${ }^{*}$ Code 7653, Naval Research Laboratory, Washington, DC 20375-5352 USA \\ ${ }^{\dagger}$ Centre de Recherche Mathématiques, Universite dé Montréal, Montréal, Canada H3C $3 \mathrm{~J} 7$
}

\begin{abstract}
González et al. (2003) have reported the discovery of an anomalous radiation component from $\approx 1-200 \mathrm{MeV}$ in GRB 941017. This component varies independently of and contains $\gtrsim 3 \times$ the energy found in the prompt $\sim 50 \mathrm{keV}-1 \mathrm{MeV}$ radiation component that is well described by the relativistic synchrotron-shock model. Acceleration of hadrons to very high energies by GRBs could give rise to a separate emission component. Two models, both involving acceleration of ultra-high energy cosmic rays with subsequent photomeson interactions, are considered. The first involves a pair-photon cascade initiated by photohadronic processes in the GRB blast wave. Calculations indicate that the cascade produces a spectrum that is too soft to explain the observations. A second model is proposed where photopion interactions in the GRB blast-wave shell give rise to an escaping collimated neutron beam. The outflowing neutrons undergo further photopion interactions to produce a beam of hyper-relativistic electrons that can lose most of their energy during a fraction of a gyroperiod in the Gauss-strength magnetic fields found in the circumburst medium. This secondary electron beam produces a hard synchrotron radiation spectrum that could explain the anomalous component in GRB 941017.
\end{abstract}

\section{INTRODUCTION}

Based on joint analysis of BATSE LAD and EGRET TASC data, González et al. (2003) recently reported the detection of an anomalous $\mathrm{MeV}$ emission component in the spectrum of GRB 941017 that decays more slowly than the prompt emission detected with the BATSE LAD in the $\approx 50-300 \mathrm{keV}$ range. The multi-MeV component lasts for $\gtrsim 200$ seconds (the $t_{90}$ duration of the lower-energy component is $77 \mathrm{sec}$ ), and is detected with the BASTE LAD near $1 \mathrm{MeV}$ and with the EGRET TASC between $\approx 1$ and $200 \mathrm{MeV}$. The spectrum is very hard, with a photon number flux $\phi(\varepsilon) \propto \varepsilon^{-1}$, where $\varepsilon=h v / m_{e} c^{2}$ is the observed dimensionless photon energy.

This component is not predicted or easily explained within the standard leptonic model for GRB blast waves, though it possibly could be related to Comptonization of reverse-shock emission by the forward shock electrons [7], including self-absorbed reverse-shock optical synchrotron radiation [11]. Another possibility is that hadronic acceleration in GRB blast waves could produce this component.

We consider two models involving acceleration of hadrons at the relativistic shocks of GRBs. In the first model, the anomalous radiation component would be explained by the pair-photon cascade radiation initiated by photohadronic processes between high-energy hadrons accelerated in the GRB blast wave and the internal synchrotron radiation field. We find, however, that the cascade radiation spectrum is too soft to be able to explain 


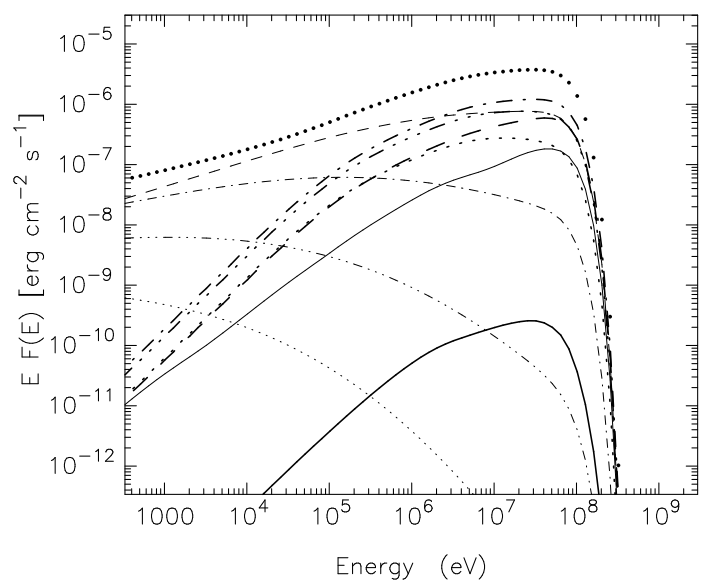

FIGURE 1. Photon energy fluence from an electromagnetic cascade initiated by photopion secondaries in a model GRB, with parameters given in the text. Five generations of Compton (heavy curves) and synchrotron (light curves) are shown. The first through fifth generations are given by solid, dashed, dotdashed, dot-triple-dashed, and dotted curves, respectively. The total cascade radiation spectrum is given by the upper bold dotted curve.

the anomalous emission component of GRB 941017. In the second model, photomeson interactions in the relativistic blast wave produce a beam of ultra-high energy neutrons, as proposed for blazar jets [2]. Photopion production of these neutrons with photons outside the blast wave produce a directed hyper-relativistic electron-positron beam in the process of charged pion decay and the conversion of high-energy photons from $\pi^{0}$ decay. These energetic leptons produce a synchrotron spectrum in the radiation reactionlimited regime extending to $\gtrsim \mathrm{GeV}$ energies, with properties in the $1-200 \mathrm{MeV}$ range similar to that measured from GRB 941017. If this model is correct, detection of this component therefore gives important indirect evidence for the acceleration of ultrahigh energy cosmic rays in GRB blast waves.

\section{HADRONIC MODELS FOR GRB 941017}

We assume that efficient proton acceleration to ultra-high energies takes place in GRB blast waves, as required in models where GRBs accelerate high-energy cosmic rays [14, 15, 3]. Photopion interactions of these protons with internal synchrotron photons and with photons from an external radiation field create neutral particles and charged pions that decay and initiate an electromagnetic cascade within the GRB blastwave.

One possible model is to attribute the anomalous component to radiation of the cascade produced within the blast wave. Fig. 1 shows the hadron-initiated cascade radiation for a model GRB at redshift $z=1$, with hard X-ray fluence $\Phi_{\text {tot }}=3 \times$ $10^{-4} \mathrm{erg} \mathrm{cm}^{-2}$, a light curve of 100 second duration divided into 50 pulses of 1 second each, and Doppler factor $\delta=100$ (see [4, 2] for more details about the model). The total amount of accelerated proton energy $E^{\prime}=4 \pi d_{L}^{2} \Phi_{t o t} \delta^{-3}(1+z)^{-1}$ is injected into the comoving frame of the GRB blast wave. 
The various generations of synchrotron and Compton radiation initiated by the cascade are shown in Fig. 1, along with the total radiation spectrum. As can be seen, the isotropic cascade radiation approaches the spectrum of an electron distribution cooling by synchrotron losses, that is, a spectral index between -1.5 and -2 . This is too soft to explain the observations of GRB 941017 with a spectral index $=-1$. Moreover, the isotropic cascade radiation should decay at the same rate as the synchrotron radiation. Thus the radiation from an electromagnetic cascade in the GRB blast wave cannot explain the anomalous component in GRB 941017.

We propose a second possibility based on our neutral beam model [2]. Ultrarelativistic neutrons formed in the reaction $p+\gamma \rightarrow n+\pi^{0}$ are not confined by the magnetic field in the GRB blastwave shell and flow out to create an energetic neutron beam. These neutrons are subject to further photopion processes with photons in the surrounding medium to form charged and neutral pions. In the Gauss-type magnetic fields surrounding GRB sources that we assume here, charged $\pi$ and $\mu$ at energies $\lesssim 10^{18} \mathrm{eV}$ decay rather than lose energy through synchrotron emission [12]. The charged pions decay into ultrarelativistic electrons and neutrinos, whereas the decay of $\pi^{0}$ produces two $\gamma$ rays that are promptly converted into electron-positron pairs on this same external radiation field. These energetic electrons (including positrons) are initially produced in the direction of the GRB jet.

The spectra of secondary electrons created by the neutron beam displays a sharp cutoff at energies $\lesssim 10^{14} \mathrm{eV}$ as a consequence of the high threshold for photomeson interactions (see Ref. [4] for more details). Electrons with Lorentz factor $\gamma$ lose energy through synchrotron radiation in an ordered magnetic field with strength $B$ at the rate $-d \gamma / d t=\sigma_{\mathrm{T}} B^{2} \gamma^{2} \sin ^{2} \psi /\left(4 \pi m_{e} c\right)$, where $\psi$ is the electron pitch angle. The corresponding synchrotron energy-loss time scale $t_{\text {syn }}=\gamma /|d \gamma / d t|$. The gyration frequency $\omega_{B}=e B / m_{e} c \gamma$, and is independent of pitch angle. When $\omega_{B} t_{s y n} \ll 1$, the electron loses almost all of its energy into synchrotron radiation in a time less than the gyroperiod. We use the term "hyper-relativistic" to refer to electrons in this radiation-reaction regime of synchrotron emission [10, 1]. The mean energy of synchrotron photons from electrons that enter the hyper-relativistic regime is independent of magnetic field (e.g., [9]).

Electrons which cool before rotating by an angle equal to the jet opening angle $\theta_{j}$ will produce synchrotron photons that are primarily directed within the jet opening angle. This condition is defined by $\omega_{B} t_{s y n} \leq \theta_{j}$, which applies to electrons with $\gamma \gtrsim \gamma_{h r}\left(\theta_{j}\right)=$ $\sqrt{4 \pi e /\left(\theta_{j} \sigma_{\mathrm{T}} B \sin ^{2} \psi\right)} \cong 3 \times 10^{7} /\left[\sin \psi \sqrt{\left(\theta_{j} / 0.1\right) B(\mathrm{G})}\right]$. Here we have taken a typical jet opening half-angle $\theta_{j}=0.1$ because this is the average value implied by the analysis leading to the standard energy reservoir result of GRBs [5]. Lower-energy electrons with $\gamma<\gamma_{h r}\left(\theta_{j}\right)$ radiate their energy over a much larger solid angle and longer time.

The characteristic synchrotron photon energy $\mathscr{E}_{\gamma}=m_{e} c^{2} \varepsilon$ radiated by electrons which lose their energy within the jet opening angle $\theta_{j}$ is

$$
\mathscr{E}_{j} \cong \frac{\hbar e B \sin \psi}{m_{e} c} \frac{\gamma_{h r}^{2}\left(\theta_{j}\right)}{(1+z)} \cong \frac{500}{\left(\theta_{j} / 0.1\right)[(1+z) / 2] \sin \psi} \mathrm{MeV}
$$

Hyper-relativistic electrons with $\gamma>\gamma_{h r}\left(\theta_{j}\right)$ rapidly lose energy through synchrotron losses and deposit all of their energy along the direction of the jet. Electrons at lower 
energies are deflected to angles $\theta>\theta_{j}$, and their emission is not seen by an on-axis observer. Hence the distribution of electrons along the jet direction always has an effective low-energy cutoff at $\gamma_{h r}\left(\theta_{j}\right)$. The production spectrum of the electrons can have an intrinsic cutoff $\gamma_{c o}$ due either to the low-energy cutoff in the escaping neutron spectrum, or to the neutron-induced photomeson secondary spectrum outside the GRB blast wave. If $\rho \equiv \gamma_{c o} / \gamma_{h r}\left(\theta_{j}\right) \geq 1$, then the observed synchrotron spectrum is a power law with -1.5 index for $\mathscr{E}_{j} \lesssim \mathscr{E}_{\gamma} \lesssim \rho^{2} \mathscr{E}_{j}$, and a photon spectrum with the same spectral index as the accelerated protons and escaping neutrons at photon energies $\mathscr{E}_{\gamma} \gtrsim \rho^{2} \mathscr{E}_{j}$ [10]. If $\rho<1$, then the observed photon spectrum at $\mathscr{E}_{\gamma} \gtrsim \mathscr{E}_{j}$ has the same spectral index as the primary neutrons.

At photon energies $\mathscr{E}_{\gamma} \ll \mathscr{E}_{j}$, the observed spectrum is produced by the same hyperrelativistic electrons with $\gamma \gtrsim \gamma_{h r}\left(\theta_{j}\right)$, but at energies $\varepsilon$ well below the peak energy $3 \gamma^{2} \varepsilon_{B}$, where $\varepsilon_{B} \equiv B / B_{c r}$ and $B_{c r}=4.41 \times 10^{13} \mathrm{G}$ is the critical magnetic field. We now derive this spectrum.

The differential energy radiated per dimensionless energy interval $d \varepsilon$ per differential solid angle element $d \Omega$ in the direction $\theta$ with respect to the direction of an electron moving with Lorentz factor $\gamma$ is given by

$$
\frac{d E}{d \varepsilon d \Omega}=\frac{e^{2}}{3 \pi^{2} \bar{\lambda}_{\mathrm{C}}}\left(\frac{\varepsilon}{\gamma \varepsilon_{B}}\right)^{2}\left(1+\gamma^{2} \theta^{2}\right)^{2}\left(\Lambda_{\|}+\Lambda_{\perp}\right),
$$

where $\bar{\lambda}_{\mathrm{C}}=\hbar / m_{e} c=3.86 \times 10^{-11} \mathrm{~cm}$ is the electron Compton wavelength, and $\Lambda_{\|}=$ $K_{2 / 3}^{2}(\xi)$ and $\Lambda_{\perp}=(\gamma \theta)^{2} K_{1 / 3}^{2}(\xi) /\left[1+(\gamma \theta)^{2}\right]$ are factors for radiation polarized parallel and perpendicular to the projection of the magnetic field direction on the plane of the sky defined by the observer's direction [8]. The factor $\xi=\varepsilon / \hat{\varepsilon}$, where $\hat{\varepsilon}=3 \varepsilon_{B} \gamma^{2} /(1+$ $\left.\gamma^{2} \theta^{2}\right)^{3 / 2}$, and $K_{n}(x)$ is a modified Bessel function of the second kind, with asymptotes $K_{n}(x) \rightarrow \frac{1}{2} \Gamma(n)(2 / x)^{n}$ in the limit $x \ll 1$, and $K_{n}(x) \rightarrow \sqrt{\pi / 2 x} \exp (-x)$ in the limit $x \gg 1$. The condition $\xi \ll 1$ corresponds to $\varepsilon \ll \hat{\varepsilon}$ where $K_{n}(\xi)$ are in their powerlaw asymptotes, and $\xi \gtrsim 1$ or $\varepsilon \gtrsim \hat{\varepsilon}$ is where $K_{n}(\xi)$ are in exponential decline. The characteristic energy $\hat{\varepsilon}$ approaches $3 \varepsilon_{B} \gamma^{2}$ when $\gamma \theta \ll 1$, and $\hat{\varepsilon}$ declines with $\theta$ according to the relation $\hat{\varepsilon} \cong 3 \varepsilon_{B} \gamma^{2} /(\gamma \theta)^{3}$ when $\gamma \theta \gg 1$. When $\varepsilon \ll \hat{\varepsilon}$, then $\Lambda_{\|} \gg \Lambda_{\perp}$ and $d E / d \varepsilon d \Omega=\left(d E_{\text {syn }} / d \varepsilon d \Omega\right) \simeq 3^{1 / 3}(1.07 e / \pi)^{2}\left(\gamma \varepsilon / \varepsilon_{B}\right)^{2 / 3} / \bar{\lambda}_{\mathrm{C}} \propto \varepsilon^{2 / 3}$. For a fixed value of $\varepsilon$, this emissivity exponentially cuts off when $\varepsilon \gtrsim 3 \varepsilon_{B} / \gamma \theta^{3}$, or when $\theta \gtrsim \theta_{\text {max }}=$ $\left(3 \varepsilon_{B} / \gamma \varepsilon\right)^{1 / 3}$.

The synchrotron emission spectrum in the limit $\mathscr{E}_{\gamma} \ll \mathscr{E}_{j}$, integrated over solid angle, is simply given

$$
\frac{d E}{d \varepsilon} \simeq 2 \pi \int_{0}^{\left(3 \varepsilon_{B} / \gamma \varepsilon\right)^{1 / 3}} d \theta \theta\left(\frac{d E_{s y n}}{d \varepsilon d \Omega}\right) \simeq \frac{3 e^{2}}{\pi \bar{\lambda}_{\mathrm{C}}} \propto \varepsilon^{0}
$$

This differs from the energy index $+1 / 3$ for the electrons in the classical regime averaged over a complete orbit because, in this case, $d \theta \sin \theta \rightarrow d \theta \sin \psi$ in the integration in eq. (3) [13]. 


\section{NEUTRON BEAMS FROM GRBS}

In our model, ultrarelativistic protons undergo photomeson interactions with the internal and external radiation photons [4], producing a beam of outflowing neutrons. Subsequent interactions of these neutrons with the external radiation field generates a beam of hyperrelativistic electrons. (The external photons could be due to pre-supernova thermal or GRB synchrotron radiations scattered by the stellar wind in the collapsar model, or by plerionic emission in the SA model.) The synchrotron radiation has a low-energy cutoff at $\mathscr{E}_{\gamma}<\mathscr{E}_{j} \sim 500 \mathrm{MeV}-1 \mathrm{GeV}$, with a specific characteristic power-law number spectral index equal to -1 , as observed for GRB 941017 [6].

The $\approx 200$ s decay time of the anomalous emission can be explained by the emission of hyper-relativistic electrons from the edges of a jet blastwave at distances $R \approx$ $10^{15}\left(\theta_{j} / 0.1\right)^{-2}[(1+z) / 2]^{-1} \mathrm{~cm}$, implying significant opacity to photomeson processes due to external photons at these distances. The differing GRB external radiation and density environments which determine the intensity of target photons could account for the unusual spectrum of GRB 941017. The neutron beam can carry up to $\sim 50 \%$ of the entire energy of injected protons [2], which can be further reprocessed about equally into neutrinos and hyper-relativistic electrons. This nevertheless requires a large nonthermal hadron-to-lepton load in the GRB blast wave in order for the fluence of the anomalous component to be comparable with that of the normal component. This large hadron load is also consistent with predictions linking cosmic rays to GRB sources [16].

Our model predicts that most of the energy of hyper-relativistic electrons will be observed at $\gtrsim 500 \mathrm{MeV}-\mathrm{GeV}$ energies as a delayed radiation component, well-suited for observations with GLAST. GRBs with anomalous $\gamma$-ray emission components should also be bright neutrino sources detectable with IceCube.

Acknowledgments. Discussions with M. González, B. Dingus, A. Königl and D. Lazzati are gratefully acknowledged. This work is supported by the Office of Naval Research and the NASA GLAST program.

\section{REFERENCES}

1. Aloisio, R., and Blasi, P. 2002, Astropar. Phys., 18, 195.

2. Atoyan, A., and Dermer, C. D. 2003, Astrophys. J., 586, 79.

3. Dermer, C. D. 2002, Astrophys. J., 574, 65.

4. Dermer, C. D., and Atoyan, A. 2003, Phys. Rev. Lett., 91, 071102.

5. Frail, D., et al. 2001, Astrophys. J., 562, L55.

6. González, M. M., Dingus, B. L., Kaneko, Y., et al. 2003, Nature, 424, 749.

7. Granot, J., and Guetta, D. 2003, Astrophys. J. Lett., 598, L11.

8. Jackson, J. D. 1975, Classical Electrodynamics (Wiley, New York).

9. de Jager, O. C., Harding, A. K., Michelson, P. F., et al. 1996, Astrophys. J., 457, 253.

10. Nelson, R. W., and Wasserman, I. 1991, Astrophys. J., 371, 265.

11. Pe'er, A., and Waxman, E. 2003, Astrophys. J. Lett., submitted (astro-ph/0310836)

12. Rachen, J. P., and Mészáros, P. 1998, Phys. Rev. D., 58, 123005.

13. Rybicki, G. B., and Lightman, A. P. 1979, Radiative Processes in Astrophysics (Wiley, New York).

14. Vietri, M. 1995, Astrophys. J., 453, 883.

15. Waxman, E. 1995, Phys. Rev. Lett., 75, 386.

16. Wick, S. D., Dermer, C. D., and Atoyan, A. 2003, submitted to Astropar. Phys. (astro-ph/0310667). 